\title{
Hybrid Capillary-Flap Valve for Vapor Control in Point-of-Care Microfluidic CD
}

T. Thio, A.A. Nozari, N. Soin, M.K.B.A. Kahar, S.Z.M. Dawal, K.A. Samra, M. Madou, and F. Ibrahim

\section{INTRODUCTION}

Microfluidics allows for the miniaturization of a complete

laboratory onto a compact disc (CD). Centrifugal

microfluidics, or microfluidic CDs, is a unique approach to

the field of microfluidics where fluids (reagents/

chemicals/clinical samples) are elegantly manipulated

by rotating specialized CDs [1]. At its core, the platform

only requires a spinning motor and disposable plastic

disc containing a fluidic network (as shown in Figure 1).

Please refer to the full text

Microfluidic CDs rely on the centrifugal force to move

fluids, uses smaller volume of reagents, and perform several

experiments in parallel, resulting in faster results at lower

costs [2]. Various fluidic functions such as mixing, decanting, etc can be automated on microfluidic CDs [3] to perform

laboratory processes ranging from blood sample

preparation to complete nucleic acid analysis [4,], [5]. Microfluidic

CDs reduces the cost, footprint, and user input of

a diagnostic platform, allowing cost-effective, portable and

automated diagnostic tools to be developed for use in pointof-

care applications. For point-of-care applications, prolonged

storage may be necessary, and the reagents/

chemicals might evaporate and mix together before

actual usage, compromising on the integrity of the result. To

complement the use of capillary valves, this paper introduces

a hybrid capillary-flap valve method that is cheap,

easy to implement, and provides segregation of vapor for 
use in point-of-care microfluidic CDs.

\section{Microfluidic VAlves}

Microfluidic valves are essential for flow control of sample

and reagents on a microfluidic $\mathrm{CD}$. There are two categories

of valves, namely passive valves, and active valves.

Passive valves have no moving parts and work on the principle

of the capillary effect [6]. Active valves on the other

hand require moving parts such as a membrane or plunger

that requires external mechanical, pneumatic, electric or

thermal force for actuation.

Capillary and Siphon Valves

Capillary valves prevent fluid flow for rotational speeds

below the burst frequency of the valve. Two kinds of passive

valves, hydrophilic and hydrophobic valves can be

designed. A hydrophilic valve can be created by having a

sudden expansion in the geometry of a channel inlet on a

hydrophilic surface [7]. A hydrophobic valve can be created

by either having a hydrophobic patch in the middle of a

channel, or having a sudden narrowing in the geometry of a

channel inlet. [8]. The figure 2 depicts these valves.

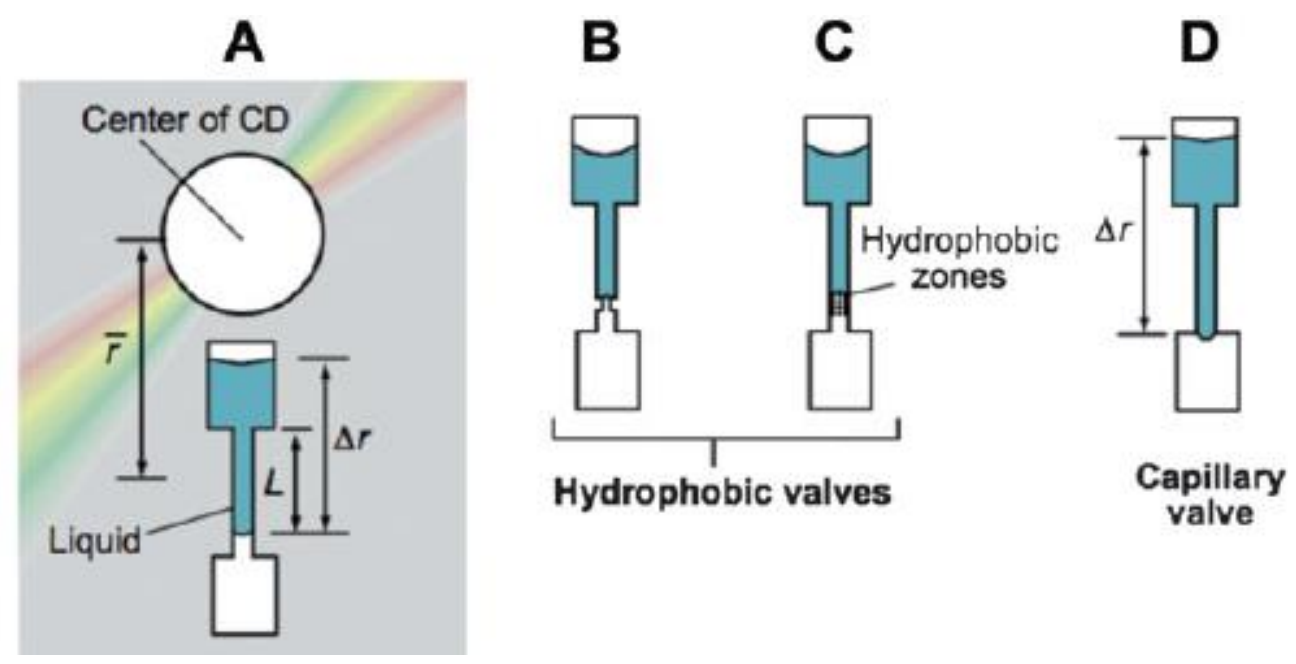

Fig. 2 Description of a microfluidic CD (A), A source and destination

chamber connected by a channel, (B) Hydrophobic valve made by a narrowing

in the channel, (C) Hydrophobic valve made by the application of 
hydrophobic material in the channel, (D) Capillary valve [8]

A siphon valve operates on the principle of the capillary

effect. Unlike the capillary valves, a siphon valve prevents

fluid flow at high rotational speeds. Figure 3 shows the

operation of a typical U-shaped siphon valve. The hydrophilic

U-shape channel between the two chambers primes

the liquid from the source to the destination chamber. At

high rotational speed, the centrifugal force pushes the liquid

outward away from the center of the $\mathrm{CD}$, trapping it at the

bottom of the U shape channel. [9] [10].

$50 \mathrm{~Hz}$
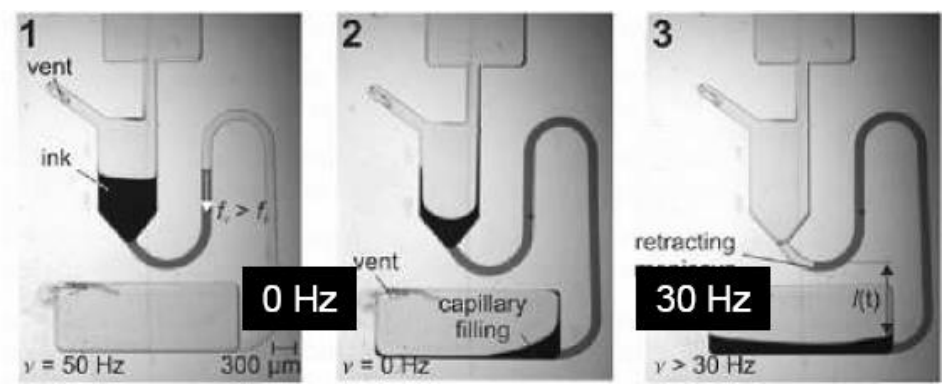

Fig. 3 Fluidic push pull through siphoning. [9] [10]

Capillary and siphon valves allow for a wide range of

applications on a microfluidic $\mathrm{CD}$, however they do not

provide segregation of vaporized liquid.

Membrane Valves

A membrane valves is constructed with a membrane

layer parallel to the channel layer [11] [12]. When pressure

is applied onto the membrane layer, the membrane deflects

and blocks the flow in the parallel channel. Figure 4 shows

a membrane type valve by Weaver et al [12]. The control

channel is below the membrane, with the flow channel

above the membrane. When pressure is applied, the membrane

deflects upward and closes off the flow channel. The

membrane valve provides flexibility in flow control, however

the fabrication process requires careful layering of the

plastic and membrane layers onto the micro device. 


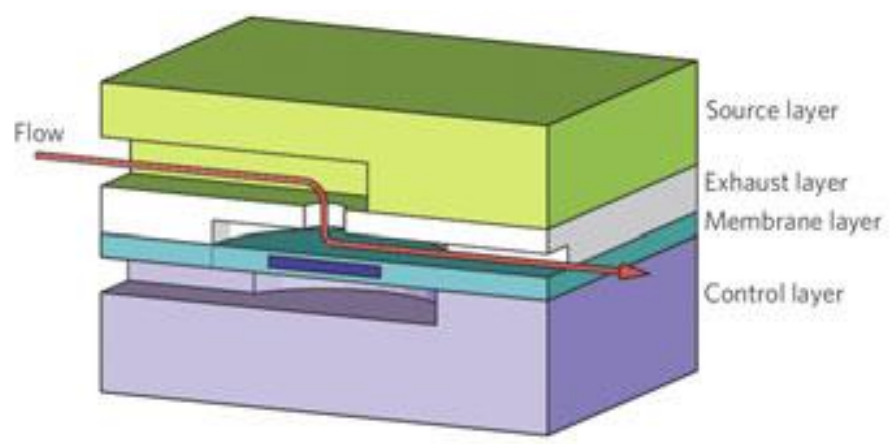

Fig. 4 The membrane valve opens when no pressure is applied [12]

Flap Valves

A flap valve is constructed by embedding a thin film within the microluific layers. Figure 5 shows a 3-way flap valve demonstrated by Mahalanabis et al [13]. The flap valve is normally closed for the 1 st channel, and normally opened for the 2 nd channel. When given an active pressure of $24.13 \mathrm{kPa}$, the flap moves down and blocks the 2 nd channel while allowing liquid to flow from the 1 st channel.

Full text is available at :

http://link.springer.com/chapter/10.1007/978-3-642-21729-6_144 